\title{
Pair-supersolid phase in a bilayer system of dipolar lattice bosons
}

\author{
C. Trefzger ${ }^{1}$, C. Menotti ${ }^{2}$, and M. Lewenstein ${ }^{1,3}$ \\ 1 ICFO - Institut de Ciencies Fotoniques, Mediterranean Technology Park, 08860 Castelldefels (Barcelona), Spain \\ ${ }^{2}$ CNR-INFM BEC and Dipartimento di Fisica, Università di Trento, I-38050 Povo, Italy \\ 3 ICREA and ICFO - Institut de Ciencies Fotoniques, \\ Mediterranean Technology Park, 08860 Castelldefels (Barcelona), Spain
}

\begin{abstract}
The competition between tunneling and interactions in bosonic lattice models generates a whole variety of different quantum phases. While, in the presence of a single species interacting via on-site interaction, the phase diagram presents only superfluid or Mott insulating phases, for long-range interactions or multiple species, exotic phases such as supersolid (SS) or pair-superfluid (PSF) appear. In this work, we show for the first time that the co-existence of effective multiple species and long-range interactions leads to the formation of a novel pair-supersolid (PSS) phase, namely a supersolid of composites. We propose a possible implementation with dipolar bosons in a bilayer two-dimensional optical lattice.
\end{abstract}

The possibility of engineering lattice models with ultra-cold gases in optical lattices is considered one of the most promising routes in the search for exotic quantum phases which escape clean demonstration in condensed matter systems. To the aim of the present work, where we show the existence of a pair-supersolid (PSS) phase, it is particularly important to briefly introduce the supersolid (SS) and the pair-superfluid (PSF) phases.

The question whether superfluidity and broken translational symmetry can coexist, leading to supersolidity, has been intriguing theoretical and experimental physicists for the last 50 years (see e.g. [1, 2, 3, 4, [5, 6]). Exact Quantum Monte Carlo simulations have demonstrated the possibility of a SS ground state in lattices [7, [8, 9, 10]. The experimental realization of a strong dipolar Chromium condensate [11] and the recent progresses towards a degenerate gas of heteronuclear polar molecules [12, 13, 14] put cold gases with long-range interaction in optical lattices among the best candidates for the creation of the SS phase [15, 16, 17].

The second important issue concerns particle vs pair condensation in Bose gases in presence of attractive interactions [18]. It has been shown [19, 20, 21, 22, 23, 24], that bosonic mixtures with inter-species attraction can actually sustain a pair-superfluid phase (PSF) without either collapsing or phase separating. The optimal candidates for the realization of the PSF phase are bosonic binary mixtures in optical lattices with interspecies interactions tunable via a Feshbach resonance [25] or, alternatively, bilayer optical lattices of dipolar particles, which create an effectively two-species system if tunneling between the two layers is suppressed [26]. The "interspecies" interaction is provided by the long-range interaction, which couples the two separate sub-systems.

However, dipolar gases offer the further opportunity of studying the combined effect of long-range interactions and inter-species coupling. In this paper, we show for the first time that the presence of both intra-layer repulsion and inter-layer attraction allows for a pair-supersolid phase (PSS), defined as a supersolid phase of composites. This phase, which joins the characteristics of the supersolid phase and the pair-superfluid phase, can be obtained in a system of dipolar bosons populating two decoupled two-dimensional (2D) optical lattice layers.

In order to demonstrate the existence of the PSS, we study the effective Hamiltonian $H_{\text {eff }}$ in the low-energy subspace of pairs, using a mean-field Gutzwiller approach and exact diagonalization. Moreover, there exist an accurate mapping at low densities of the effective Hamiltonian onto the single component extended Hubbard model [7]; the fact that the latter model supports SS implies the existence of PSS in our case.

We consider polarized dipolar particles in two decoupled 2D layers (see Fig. 10. This geometry can be obtained by using anisotropic optical lattices or superlattices, which can exponentially suppress tunneling in one direction. The in-plane dipolar interaction is isotropic and repulsive. The inter-layer interaction depends on the relative position between the two dipoles, but is dominated by the nearest neighbor attractive interaction $W<0$ between two atoms at the same lattice site in different layers. In the following, we include only nearest

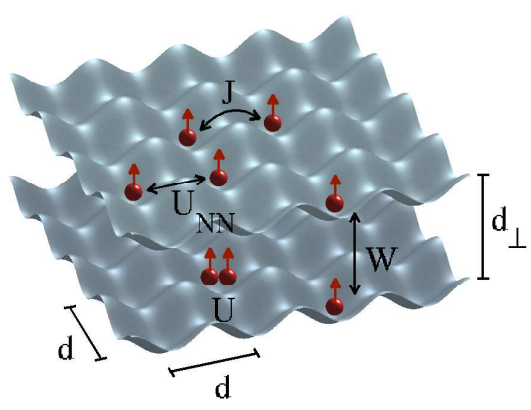

FIG. 1: Schematic representation of two 2D optical lattice layers populated with dipolar bosons polarized perpendicularly to the lattice plane. The particles feel repulsive on-site $U$ and nearest neighbor $U_{\mathrm{NN}}$ interactions. Inter-layer tunneling is completely suppressed, while a nearest neighbor inter-layer attractive interaction $W$ is present. 
neighbor in-plane $\left(U_{\mathrm{NN}}\right)$ and out-of-plane $(W)$ dipolar interactions. The relative strength between $U_{\mathrm{NN}}$ and $W$ can be tuned by changing the spacing $d_{\perp}$ between the two layers, relative to the $2 \mathrm{D}$ optical lattice spacing $d$. Due to the dependence of the dipole-dipole interaction like the inverse cubic power of the distance, the ratio $|W| / U_{\mathrm{NN}}$ can be tuned over a wide range. While it can be negligible for $d_{\perp} \gg d$ making the system asymptotically similar to a single $2 \mathrm{D}$ lattice layer, it can also become relevant and give rise to interesting physics, not existing in the single layer model [26, 27, 28, 29, 30, 31, 32].

We start from the generalized extended Bose-Hubbard Hamiltonian

$$
\begin{aligned}
H= & \sum_{i, \sigma}\left[\frac{U}{2} n_{i}^{\sigma}\left(n_{i}^{\sigma}-1\right)+\sum_{\langle j\rangle_{i}} \frac{U_{\mathrm{NN}}}{2} n_{i}^{\sigma} n_{j}^{\sigma}-\mu n_{i}^{\sigma}\right]+ \\
& +\sum_{i} W n_{i}^{a} n_{i}^{b}-J \sum_{\langle i j\rangle}\left[a_{i}^{\dagger} a_{j}+b_{i}^{\dagger} b_{j}\right]
\end{aligned}
$$

where $\sigma=a, b$ indicates the two species (which in the specific case considered here are atoms in the lower and upper 2D optical lattice layer respectively), $U$ is the on-site energy, $U_{\mathrm{NN}}$ the intra-layer nearest neighbors repulsion, $W$ the inter-layer attraction, $J$ the intra-layer tunneling parameter and $\mu$ the chemical potential. The parameters $U$ and $J$ are equal for the upper and lower layers and the chemical potentials $\mu$ are the same, since equal densities in the two layers are assumed. The symbols $\langle j\rangle_{i}$ and $\langle i j\rangle$ indicate nearest neighbors.

The PSS is characterized by vanishing single particle order parameters $\langle a\rangle=\langle b\rangle=0$, and non vanishing pair order parameter $\langle a b\rangle \neq 0$, coexisting with broken translational symmetry, namely a modulation of both density and order parameter on a scale larger than the one of the lattice spacing, analogously to the supersolid phase. The physics leading to the formation of composites relies on second order tunneling and takes place in the low-energy subspace where single-particle hopping is suppressed. The theoretical description of the PSS phase cannot be based on standard mean-field theory, which accounts for particle hopping through the replacement in the Hamiltonian of the single particle creation and destruction operators by their expectation values, because in this approximation second order tunneling completly vanishes.

A successful way to account for second order tunneling is to write an effective Hamiltonian in the subspace of pairs and include tunneling through second order perturbation theory [19, 20, 21, 33]. The validity of the effective Hamiltonian relies on the existence of a low-energy subspace well separated in energy from the subspace of virtual excitations, to which it is coupled via single particle hopping. Such second order couplings are related to the super-exchange interaction, recently measured in a series of double-well systems [34].

The low-energy subspace of pairs is spanned by all classical distributions of atoms in the lattice $|\alpha\rangle=\prod_{i}\left|n_{i}, n_{i}\right\rangle$ with equal occupation of the two species $a$ and $b$. For $(U+W) / U \rightarrow 0$, asymptotically all classical states $|\alpha\rangle$ become stable with respect to single particle-hole excitations and develop an insulating lobe at finite $J$. The energy of single particle-hole excitations is of the order of $U$ at $J=0$ and is given by the width of the lobes at finite $J$ (see e.g. thin blue lobes in Fig 2). This situation has to be compared to the single layer situation $(W=0)$ with nearest neighbor interactions, where only uniform Mott phases and checkerboard insulating phases are stable. The relevant virtual subspace is obtained from the states $|\alpha\rangle$ by breaking one composite, namely $\left|\gamma_{i j}^{(a)}\right\rangle=a_{i}^{\dagger} a_{j}|\alpha\rangle / \sqrt{n_{j}\left(n_{i}+1\right)}$ and $\left|\gamma_{i j}^{(b)}\right\rangle=b_{i}^{\dagger} b_{j}|\alpha\rangle / \sqrt{n_{j}\left(n_{i}+1\right)}$.

In the pair-state basis, the matrix elements of the Hamiltonian in second order perturbation theory are given by

$$
\begin{aligned}
& \left\langle\alpha\left|H_{\mathrm{eff}}\right| \beta\right\rangle=\left\langle\alpha\left|H_{0}\right| \beta\right\rangle+ \\
& -\frac{1}{2} \sum_{\gamma}\left\langle\alpha\left|H_{1}\right| \gamma\right\rangle\left\langle\gamma\left|H_{1}\right| \beta\right\rangle\left[\frac{1}{E_{\gamma}-E_{\alpha}}+\frac{1}{E_{\gamma}-E_{\beta}}\right],
\end{aligned}
$$

where $H_{0}$, given by the interaction terms of the Hamiltonian (1), is diagonal on the states $|\alpha\rangle$, and the single particle tunneling term $H_{1}=-J \sum_{\langle i j\rangle}\left[a_{i}^{\dagger} a_{j}+b_{i}^{\dagger} b_{j}\right]$ is treated at second order.

For a given state $|\alpha\rangle$,

$$
E_{\gamma_{i j}}-E_{\alpha}=U+(U+W)\left(m_{i}-m_{j}\right)+U_{\mathrm{NN}} \Delta m_{\mathrm{NN}}^{i j},
$$

with $\Delta m_{\mathrm{NN}}^{i j}=\sum_{\langle k\rangle_{i}} m_{k}-\sum_{\langle k\rangle_{j}} m_{k}-1$, where $m_{i}$ indicates the pair occupation number at site $i$. For $\mathrm{U}+\mathrm{W}, U_{\mathrm{NN}} \ll U$, the denominators $E_{\gamma_{i j}}-E_{\alpha}$ are all of order $U$, which leads to

$$
H_{\mathrm{eff}}^{(0)}=H_{0}-\frac{2 J^{2}}{U} \sum_{\langle i j\rangle}\left[m_{i}\left(m_{j}+1\right)+c_{i}^{\dagger} c_{j}\right],
$$

where $c_{i}$ and $c_{i}^{\dagger}$ are the pair destruction and creation operators such that $c_{i}^{\dagger}\left|m_{i}\right\rangle=\left(m_{i}+1\right)\left|m_{i}\right\rangle$. One can easily obtain corrections to $H_{\mathrm{eff}}^{(0)}$ by expanding (3) at higher orders in $(U+W) / U$ and $U_{\mathrm{NN}} / U$.

In this work, we provide a mean-field solution to effective Hamiltonian (4). We perform a perturbative treatment at first order in the pair order parameter $\psi=\langle c\rangle$ to determine the boundaries of the insulating lobes. Furthermore, we solve the time dependent Gutzwiller equations in imaginary time to determine the nature of the superfluid phases outside the insulating lobes. We point out that in spite of its simplicity, the mean-field treatment of the effective Hamiltonian is able to include important features like the re-entrant behavior of the lobes (as predicted by exact t-DMRG calculations for the 1D geometry in [26]). 


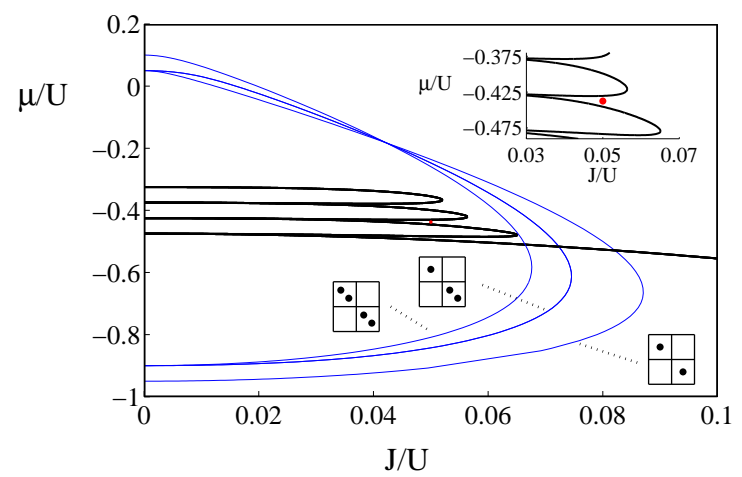

FIG. 2: Pair insulating lobes for $\nu=0,1 / 2,1,3 / 2$ (thick lines); Lobes with respect to single particle-hole excitations (thin blue lines) for the dominant configurations in the ground state at $J=0.05 U$ and $\mu=-0.4375 U$, namely $m_{i}=0$ and $m_{j}=1,2$ (for $i, j$ nearest neighboring sites). The inset shows a zoom of the pair phase diagram.

For any non-zero $W<0$, the lowest possible excitations on top of a classical configuration of pairs are obtained by adding (removing) one pair at site $i$. The energy costs, respectively given by $E_{\mathrm{P}}^{i}(J)=-2 \mu+$ $2 U m_{i}+\left(2 m_{i}+1\right) W+2 V_{\text {dip }}^{1, i}-\left(2 J^{2} / U\right) \sum_{\langle k\rangle_{i}}\left(2 m_{k}+1\right)$ and $E_{\mathrm{H}}^{i}(J)=2 \mu-2 U\left(m_{i}-1\right)-\left(2 m_{i}-1\right) W-2 V_{\text {dip }}^{1, i}+$ $\left(2 J^{2} / U\right) \sum_{\langle k\rangle_{i}}\left(2 m_{k}+1\right)$, depend quadratically on the tunneling coefficient $J$. In the previous expressions, $V_{\text {dip }}^{1, i}>0$ is the dipole-dipole interaction of one atom placed at site $i$ with the rest of the particles belonging to the same layer. The mean-field order parameters $\psi_{i}$ satisfy

$$
\psi_{i}=\frac{2 J^{2}}{U}\left[\frac{\left(m_{i}+1\right)^{2}}{E_{\mathrm{P}}^{i}(J)}+\frac{m_{i}^{2}}{E_{\mathrm{H}}^{i}(J)}\right] \bar{\psi}_{i},
$$

with $\bar{\psi}_{i}=\sum_{\langle k\rangle_{i}} \psi_{k}$ being the sum of the nearestneighbour order parameters. Using Eq. (5), one can calculate the mean-field lobes for any given configuration of pairs in the lattice. The lobes for the checkerboard and doubly occupied checkerboard are shown in Fig. 3 for the 0th (full lines) and 1st order (dashed lines) effective Hamiltonians. The comparison between the two shows that, for the parameters considered here, the 0th order already captures the physics accurately. The $J^{2}$ dependence of the energy of the elementary excitations is at the origin of the re-entrant behavior of the lobes.

Based on the Gutzwiller Ansatz for the pair wavefunction $|\Phi\rangle=\prod_{i} \sum_{m} f_{m}^{(i)}|m, i\rangle$, we predict the existence of three different phases: insulating phases, PSF and PSS. For $U+W<z U_{\mathrm{NN}}$, being $z$ the number of nearest neighbors, the insulating phases show checkerboard ordering not only at filling factor $\nu=1 / 2$, but also at filling factor $\nu=1$. Outside the insulating lobes, depending on density and tunneling, we find either PSF or PSS. The stability analysis of the PSS phase against phase separation is beyond our mean-field treatment. However, for small $U+W<z U_{\mathrm{NN}}$, by doping the checkerboard above half filling, the extra pair goes on an already occupied site and the analogy to the single-species extended BoseHubbard model [7] suggests that the system stabilizes to a PSS phase. Instead, the PSS phase at density lower than $1 / 2$ (which our mean-field treatment predicts only in a very small region close to the tip of the $\nu=1 / 2$ lobe) should be unstable towards phase separation. Nevertheless, the inclusion of more neighbors to the in-plane dipolar interaction is expected to remove this instability [35].

To get reliable results, one should combine the Gutzwiller predictions with an estimate of the limits of validity of $H_{\mathrm{eff}}^{(0)}$, beyond which the subspace of pairs looses its meaning. For each point of the phase diagram, from the ground state Gutzwiller wavefunction, we select the dominant classical configurations with the criteria $\left|f_{m}^{(i)}\right|^{2}>0.05$ and $\left|\prod_{i} f_{m}^{(i)}\right|>0.02$ [36] and calculate for each of these configurations, the lobe with respect to single particle-hole excitations. If the system at this given point of the phase diagram turns out to be stable against all dominant single particle-hole excitations (in other words, if this point is inside all selected single particle-hole lobes), $H_{\mathrm{eff}}^{(0)}$ is considered valid at that point. This procedure is shown for $J=0.05 U$ and $\mu=-0.4375 U$ in Fig. 2, In Fig. 3, we show the resulting phase diagram for $U_{\mathrm{NN}}=0.025 U$ and $W=-0.95 U$. The shaded area represents the region of PSS, compatible with the above validity conditions for $H_{\text {eff }}^{(0)}$. Interestingly, the $\prec$-like shape of the allowed pairing regions (PSF and PSS) matches the ones found in [26].

The existence of the PSS phase is supported also by the exact diagonalization of $H_{\text {eff }}^{(0)}$ for up to 6 pairs on a $2 \times 8$ lattice with periodic boundary conditions. At small $J$, the ground state is almost doubly degenerate. This can correspond to two insulating checkerboards (for half filling) or two checkerboard supersolids shifted by one lattice constant, with a large gap to excited states. This double degeneracy, together with a finite measure of the coherence, provided by a non vanishing expectation value of the tunneling term, are indications of the existence of the PSS. At larger $J$, the quasi-degeneracy of the two lowest eigenstates disappears and the ground state becomes well separated in energy from all excited states, indicating the crossover to the PSF. It is important to remark that in the absence of nearest-neighbor interaction, the ground state is always non degenerate and no signature for the PSS is ever found.

Finally, we observe that, upon appropriate renaming of the parameters, $H_{\mathrm{eff}}^{(0)}$ (4) can be mapped onto the Hamiltonian used in [7] to demonstrate the existence of the 


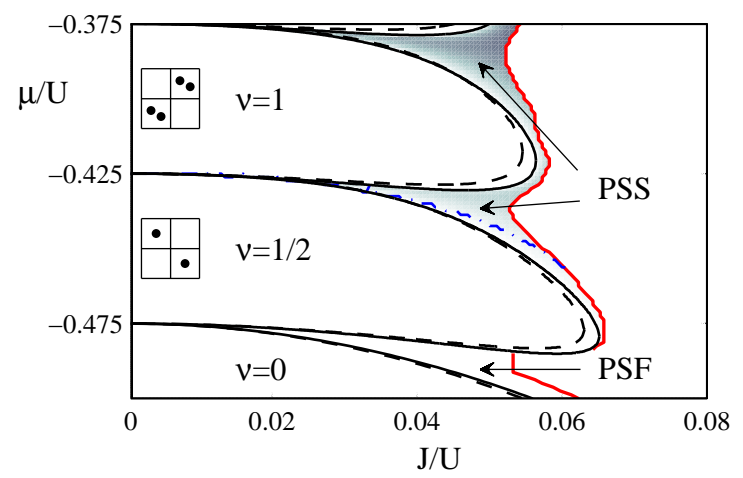

FIG. 3: Phase diagram of the effective Hamiltonian, with $U_{\mathrm{NN}}=0.025 U, W=-0.95 U$, which can be obtained for $d_{\perp}=0.37 d$. The black full lines are the semi-analytic solution of Eq. (5) indicating the boundaries of the insulating lobes for the checkerboard $(\nu=1 / 2)$ and the doubly occupied checkerboard $(\nu=1)$. The black dashed lines are the boundaries of the insulating lobes for 1st order expansion of $H_{\text {eff }}$. The shaded area is the PSS phase predicted by the Gutzwiller approach. The red line indicates the estimated limit of validity of $H_{\mathrm{eff}}^{(0)}$. The blue dash-dotted line indicates the upper limit of the region where the mapping onto [7] is almost exact.

SS phase for soft core bosons with nearest neighbor interactions. Due to the different action of the tunneling terms, namely $\left\langle n_{i}+1, n_{j}-1\left|a_{i}^{\dagger} a_{j}\right| n_{i}, n_{j}\right\rangle=\sqrt{\left(n_{i}+1\right) n_{j}}$ in [7] and $\left\langle m_{i}+1, m_{j}-1\left|c_{i}^{\dagger} c_{j}\right| m_{i}, m_{j}\right\rangle=\left(m_{i}+1\right) m_{j}$ in our case, the mapping of the two Hamiltonians is exact when only the number states $m=0,1$ are populated. Under this condition, the results of 7] translate to the existence of the PSS in our problem. The region below the dash-dotted blue line in Fig. 3, which corresponds to $\left|f_{0}^{(i)}\right|^{2}+\left|f_{1}^{(i)}\right|^{2}>0.9, \forall i$, namely a close to exact mapping, includes part of the PSS region.

Summarizing, we have studied the phase diagram of a bilayer system of 2D dipolar lattice gases, in the limit of close layers, and demonstrated the existence of a novel PSS phase, namely a supersolid phase of pairs. The existence of the PSS phase has been previously discussed for anisotropic $t-J$ models 37], but no evidence of it has been found. However, the Hamiltonian we discuss in the present work differs from the anisotropic $t-J$ spin Hamiltonian in three crucial respects, all of which should favor the existence of the PSS phase: (i) it deals with softcore bosons (vs. hard-core); (ii) it considers on-site interspecies attraction (vs. nearest-neighbor inter-species attraction); (iii) it includes nearest-neighbor intra-species repulsion. For these reasons, we believe that the existence of the PSS phase will be confirmed by exact calculations, also beyond the limits of validity of our effective mean-field approach.

We acknowledge support of Spanish MEC (FIS200800784, QOIT), EU projects SCALA and NAMEQUAM, ERC grant QUAGATUA. The authors thank O. Dutta, M. Modugno, L. Santos, S. Stringari, B. Svistunov, for interesting discussions.
[1] N. Prokof'ev, Advances in Physics, Volume 56 Issue 2, 381 (2007), arXiv:cond-mat/0612499.

[2] E. Kim and M.H.W. Chan, Nature 427, 225 (2004); Science 305, 1941 (2004); Phys. Rev. Lett. 97, 115302 (2006).

[3] L. Pollet et al., Phys. Rev. Lett. 98, 135301 (2007).

[4] B. Svistunov, Phys. A: Cont. Mat. 404, 521 (2009).

[5] S. Sasaki et al., Science 313, 1098 (2006)

[6] M. W. Ray and R. B. Hallock Phys. Rev. Lett. 100, 235301 (2008).

[7] P. Sengupta et al., Phys. Rev. Lett. 94, 207202 (2005).

[8] G.G. Batrouni and R.T. Scalettar, Phys. Rev. Lett. 84, 1599 (2000).

[9] F. Hébert et al., Phys. Rev. A 65, 014513 (2001)

[10] Y.-C. Chen et al, Phys. Rev. B 77, 014524 (2007)

[11] T. Lahaye et al., Nature 448, 672 (2007).

[12] J.G. Danzl et al., Science 321, 1062 (2008).

[13] K.-K. Ni et al., Science 322, 231-235 (2008)

[14] S. Ospelkaus et al., arXiv:0811.4618.

[15] K. Góral et al, Phys. Rev. Lett. 88, 170406 (2002).

[16] D.L. Kovrizhin et al., Europhys. Lett. 72, 162 (2005).

[17] V.W. Scarola et al., Phys. Rev. A 73, 051601(R) (2006).

[18] P. Nozières and D. Saint James, J. Physique 43, 1133 (1982).

[19] E. Altman et al., New J. Phys. 5, 113 (2003).

[20] A.B. Kuklov and B.V. Svistunov, Phys. Rev. Lett. 90,
100401 (2003).

[21] A. Kuklov et al., Phys. Rev. Lett. 92, 050402 (2004).

[22] S..G. Söyler et al., arXiv:0811.0397

[23] A. Hubener el at., arXiv:0902.2212

[24] B. Paredes and J.I. Cirac, Phys. Rev. Lett. 90, 150402 (2003).

[25] J. Catani et al., Phys. Rev. A 77, 011603(R) (2008).

[26] A. Argüelles and L. Santos, Phys. Rev. A 75, 053613 (2007).

[27] G.-H. Chen and Y.-S. Wu, Phys. Rev. A 67, 013606 (2003).

[28] D.-W. Wang et al., Phys. Rev. Lett. 97, 180413 (2006).

[29] Daw-Wei Wang, Phys. Rev. Lett. 98, 060403 (2007).

[30] S. Yi et al., Phys. Rev. Lett. 98, 260405 (2007).

[31] D.-W. Wang and E. Demler, arXiv:0812.1838

[32] M. Klawunn and L. Santos, arXiv:0812.3543

[33] see e.g. Atom-Photon interactions, C. Cohen-Tannoudji, J. Dupont-Roc, and G. Grynberg, Wiley (1998).

[34] S. Trotsky et al., Science 319, 295 (2008).

[35] B. Capogrosso-Sansone et al., arXiv:0906.2009.

[36] We have checked that the validity region is not strongly modified upon small changes in these conditions.

[37] M. Boninsegni and N.V. Prokof'ev, Phys. Rev. B 77, 092502 (2008). 